\title{
A Two-Product Inventory Model with a Joint Ordering Policy
}

\author{
A. Masache \\ Department of Statistics and Operations Research, National University of Science and Technology, \\ P.O. Box AC 939 Ascot, Bulawayo, Zimbabwe \\ Correspondence should be addressed to A. Masache; amon.masache11@gmail.com
}

Received 15 April 2013; Accepted 12 October 2013

Academic Editor: Khosrow Moshirvaziri

Copyright (C) 2013 A. Masache. This is an open access article distributed under the Creative Commons Attribution License, which permits unrestricted use, distribution, and reproduction in any medium, provided the original work is properly cited.

Inventory is one of the most visible and tangible aspects of doing business. This is the reason why most problems of a business often end up in inventory. Most inventory studies focus on a single type of product or item such that optimality decisions are arrived at for that single product. In this study, we create a scenario where two types of products are considered in such a way that there exists a joint ordering policy. A continuous review inventory model was developed but ended up with a single period review model for simplicity reasons. We tried to exhaust all main cost elements and fed them into the model in addition to investigating the possible limitations that may constrain the whole decision making process. Six constraints were found and a mathematical programming model was developed. We further went on to prove that the mathematical inventory-programming model developed minimises the total inventory cost in such a scenario.

\section{Introduction}

The role of inventory management is to coordinate the actions of sales, marketing, production, and purchasing to ensure that the correct level of stocks is held to satisfy customer demand at the lowest possible costs; hence, aiming to balance out supply and demand by regulating the supply of goods in such a way that they match demand conditions as close as possible. Some demand conditions require the application of multiple replenishment orders in continuousreview inventory systems. Reference [1] highlighted that in such a study the concept of common demand for some products arises; for example, when a customer arrives at a shop that sells two brands of soft drinks, the customer would be satisfied by a particular brand with a certain probability. Also, when a supplier is the same for several products under consideration, the retailer will prefer to have simultaneous replenishment for all products due to several reasons like cost considerations and so forth.

Researchers like [2] allowed shortages and assumed that the lost sale policy could be applied when studying such kind of demand conditions. Their demand was not deterministic but stochastic, following a Poisson distribution. Later, [3] developed optimal coordination policies for manufacturing and supply divisions. The authors considered a problem where the responsibility of the manufacturing division was to provide a sufficient amount of raw materials so that the required production level could be achieved. Reference [4] studied such demand conditions and it was under continuous review of multiproduct inventory systems. Like Mohebi and Posner [2], they also assumed that demand follows a Poisson distribution and that lead time has a probability distribution function. The results obtained were placements of reorders, lost demands, and replenished units after analysing the imbedded renewal process describing the system. Reference [5] took the EOQ model and extended it to joint replenishment policy for purchasing expensive raw materials. Their cost elements were holding cost, purchasing cost under incremental discount, clearance and fixed order cost, transportation cost, and costs for the next order. Earlier on, a similar problem of joint coordination had been studied by [1]. Instead, the problem was between manufacturing and supplying divisions in a short-life cycle multiproduct environment. In their study, they did not consider a continuous review multiproduct inventory system on which our study focuses. Elsewhere, coordinated inventory strategies for items in each cluster were determined using Genetic stepwise clustering and statistical agglomerative clustering methods [6]. 
Just like on the study done by [5], they incorporated an EOQ model, however, into a multi-item multibranch firm with an aim of finding optimal ordering and warehousing strategies. Indeed, their mixed inventory policy with two clustering approaches had more savings than the pure inventory policy. Recently, [7] extended the study on a joint replenishment problem to Pareto analysis by grouping the items under consideration to an $\mathrm{ABC}$ classification. They concluded that a continuous review can be carried on the sum of the demands for products in the A classification.

In addition to the field of inventory modeling, in this paper, we attempt to develop a joint ordering model on two products with deterministic demands but in a continuous review period. The study focuses on a priority product (the first product) in such a way that most cost elements considered are for the first product. The model becomes a joint ordering policy basically in the sense that the first product cannot be ordered without considering the inventory levels of the second product. In addition, we try to explore some limitations that may be encountered on implementing joint ordering policy thereby coming up with a nonlinear inventory programming model.

\section{Assumptions}

The following assumptions are considered in this study.

(i) There is no backlogging on all products.

(ii) Holding cost of product 1 due to the presence of product 2 or vice versa is equal to the holding cost of product 1.

(iii) Product 1 has got a higher demand than product 2 or the rate at which product 1 depletes is higher than that of product 2. As a result, it is nearly impossible to experience product 2 stockouts.

(iv) Demand for product 1 during its stock-out time can be satisfied by product 2 . That is, in the presence of the two products most customers prefer product 1 to product 2 . However, if product 1 is out of stock, then customers can still buy product 2 as a substitute for product 1 .

(v) Both products are always ordered at the same time and when product 1 is completely depleted.

(vi) Both products have got the same lead time (but due to joint ordering of products with different depletion rates in different periods, order quantities will be different in those periods) and when the reorder materialises, inventory levels always reach a maximum.

(vii) Storage facilities are always in proper conditions.

\section{Model Development}

3.1. Notations. The problem is presented as an optimality problem and the following notations are used.

$x_{i}(t)$ : Inventory levels for product $i(i=1,2)$ at time $t$

$x_{i}^{*}(t)$ : Optimal inventory level for product $i$ at time $t$

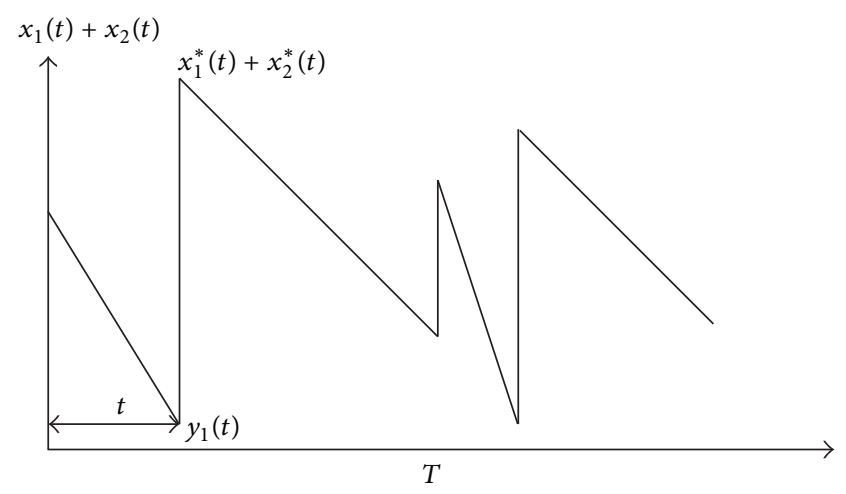

FIGURE 1: Variations of inventory level with time.

$x_{i j}(t)$ : Product $i$ availability in the absence of product $j$ at time $t$, for $i, j=1,2$ and $i \neq j$

$\mu(t)$ : Joint ordering rate at time $t$

$\mu_{i}(t)$ : Product $i$ ordering quantity at time $t$

$\mu_{i}^{*}(t)$ : Optimal ordering rate for product $i$

$T$ : Planning period length

$c_{i}$ : Ordering cost coefficient for product $i$

$h_{i}$ : Holding cost coefficient for product $i$

$y_{i}(t)$ : Minimum inventory level of product $i$ at time $t$

$D_{i}(t)$ : The periodic demand of product $i$ at time $t$

$L(t)$ : The periodic lead time such that $L$ becomes the periodic independent lead time.

If we define the optimal inventory levels as the stock level that ensures that the system does not experience any stockout of both products, then the diagrammatic illustration is as shown in Figure 1 above.

Thus the economic order quantity of product $i$ at time $t$ becomes

$$
\begin{gathered}
\qquad u_{i}(t)=\sqrt{\frac{2 D_{i}(t)}{h_{i}}}, \\
\text { Optimal ordering rate }=\frac{\text { Demand }}{\operatorname{Re} \text { order level }},
\end{gathered}
$$

where Re order level $=$ average daily demand $\times$ lead time, that is;

$$
\mu_{i}^{*}(t)=\frac{D_{i}(t)}{\left(x_{i}^{*}(t) / t\right) L(t)} .
$$

Thus, the minimum stock level for product $i$ at time $t$ will be given by the reorder level subtracted from the average usage for a normal planning period.

3.2. The Cost Function. There is always a penalty charged for holding stock above or below the optimal stock levels at any period. Thus, the cost incurred for holding more or less stocks than expected is the holding cost multiplied by the modulus 
of the inventory level for stock $i$ at time $t$ less than the optimal inventory level for stock $i$. That is, this cost factor can be expressed as $h_{i}\left(x_{i}(t)-x_{i}^{*}(t)\right)^{2}$. A penalty that can be charged is for placing more orders than expected per planning period. That cost factor can also be expressed as $\mu_{i}(t)\left(c_{i}-\mu(t)\right)$. In addition, the holding cost of the availability stock $i$ in the absence of stock $j$ can be incurred, and it is written as $h_{i} x_{i j}$ (where $i \neq j$ for $i, j=1,2$ ). Now we can recognise the total inventory cost for one product as the sum of the identified costs such that

$$
\begin{aligned}
\text { Total cost }= & \text { holding cost }+ \text { ordering cost } \\
& + \text { holding cost incurred when stock } i \\
& \text { is available in the absence of } j .
\end{aligned}
$$

The combined total inventory cost of the two products is now found by summing up the separate total cost and integrating over the planning periods:

$$
\begin{aligned}
C=\int_{0}^{T}\left\{\sum_{i=1}^{2} h_{i}\left(x_{i}(t)-x_{i}^{*}(t)\right)^{2}\right. & \\
& \left.+\mu_{i}(t)\left(c_{i}-\mu(t)\right)+h_{i} x_{i j}(t)\right\} d t .
\end{aligned}
$$

It has to be noted that ordering at different cycles is done at different stock levels of the nonpriority product (ordering is done when the priority product has completely depleted) because in different cycles there would be different demands $\left(D_{i}(t)\right)$. That is, depletion rates for both products are time dependent or are different in different cycles. As a result, ordering costs always depend on the periodic cycle when the order is placed. Now, investigating the inventory problem under $T$ continuous review periods proves to be a complicated task. As a result, we can simplify the problem by studying the problem within a single period review. That is, the integrating aspect is removed from (2) above and all timedependant variables become constants with respect to time:

$$
C=\sum_{i=1}^{2} h_{i}\left(x_{i}-x_{i}^{*}\right)^{2}+\mu_{i}\left(c_{i}-\mu\right)+h_{i} x_{i j}
$$

Its is important to note that the previous equation is a cost function, so, it is only logical to minimise the function. In Section 4 we will prove that (5) is indeed a minimisation problem.

3.3. Constraints. If $x_{j}$ cannot be ordered without $x_{i}$ (though a customer can buy product $j$ when desiring product $i$ which is out of stock, for $i \neq j$ ), then the optimal inventory level for product $i$ must be the level to meet the normal demand to a certain level at any given time as well as for the sudden rise in stock $i$ demand. Thus, the optimal stock level for stock $i$ should be equal to or more than "\{inventory level for stock $i$ at time $t$ plus the product $i$ ordered quantity at time $t\}$ minus \{demand at time $t$ of stock $i$ plus stock $i$ issued in the absence of stock $i\}$."
That is,

$$
\begin{array}{r}
x_{i}^{*}(t) \geq x_{i}(t)+\mu_{i}(t)-x_{i j}(t)-D_{i}(t), \\
(i, j=1,2, i \neq j) .
\end{array}
$$

If we assume that product $i$ is the main product, then stock of product $i$ is always more than stock of product $j$ at any given time $t$ and must always be at least the same level with the inventory level for product $j$. That is, the optimal inventory level for product $i$ is always at least that of product $j$. Since both stocks are reordered when the inventory level reaches the respective reorder levels, then we can assume that when reorder materializes, inventory levels of each stock are brought to their maximum level. But if inventory level of stock $i$ reaches its reorder level and stock $j$ still at its maximum level or not yet reached its reorder level, then only stock $i$ is ordered. Thus, the joint orders in any period must be at most the square root of the two optimal ordering rates.

That is,

$$
u(t) \leq \sqrt{\mu_{i}^{*}(t) \mu_{j}^{*}(t)}, \quad(i \neq j)
$$

We can write the optimal ordering rates in terms of their respective optimal inventory levels so that we can deal with the decision variables. That is,

$$
\begin{aligned}
u(t) & \leq \sqrt{\left(\frac{t D_{i}(t)}{x_{i}^{*}(t) L(t)}\right)\left(\frac{t D_{j}(t)}{x_{j}^{*}(t) L(t)}\right)} \\
& \leq \sqrt{\frac{1}{x_{i}^{*}(t)} \cdot \frac{1}{x_{j}^{*}(t)} \cdot \frac{t^{2} D_{i}^{2}(t)}{L^{2}(t)}}
\end{aligned}
$$

(common demand condition applied)

$$
=\sqrt{\prod_{i=1}^{2} \frac{D_{i}(t)}{x_{i}^{*}(t)} \frac{t}{L(t)}} .
$$

Therefore, joint ordering constraint can be written as follows:

$$
\mu^{2}(t) \leq \prod_{i=1}^{2} \frac{D_{i}(t)}{x_{i}^{*}(t)} \frac{t}{L(t)}
$$

At all times, the inventory level for stock $i$ must not be less than the minimal inventory level in order to avoid loss of sales. This means that it is not possible for the inventory level of stock $j$ to exceed that of stock $i$ at any particular time. In addition, inventory levels for both stocks $i$ and $j$ at any time can neither be less than zero nor exceeding the holding capacity of the storage facility. That is, the capacity of the storage facility becomes $x_{i}(t)+x_{j}(t)$. If we let $H$ be the holding capacity and $I_{\min }=\left(y_{i}(t)+y_{j}(t),(i \neq j)\right)$ be the minimum inventory allowed in the facility, then

$$
I_{\min } \leq x_{i}(t)+x_{j}(t) \leq H
$$


3.4. Model Description. Combining the cost function and associated constraints will now give us the following nonlinear mathematical inventory-programing model for a single planning period review:

$$
\operatorname{Min} C=\sum_{i=1}^{2} h_{i}\left(x_{i}-x_{i}^{*}\right)^{2}+\mu_{i}\left(c_{i}-\mu\right)+h_{i} x_{i j}
$$

Subject to

$$
\begin{gathered}
x_{i}^{*} \geq x_{i}+\mu_{i}-x_{i j}-D_{i}, \\
\mu^{2} \leq \prod_{i=1}^{2} \frac{D_{i}}{x_{i}^{*}} \cdot \frac{T}{L}, \\
x_{i}^{*}>x_{j}^{*}, \\
I_{\min } \leq x_{i}^{*}+x_{j}^{*} \leq H, \\
x_{i}^{*}, x_{j}^{*} \geq 0, \mu \geq 0, i \neq j, i, j=1,2 .
\end{gathered}
$$

\section{Problem Classification}

In order for us to classify the above nonlinear inventoryprogramming problem, we have to consider the Hessian matrix $\left(H\left(X^{*}\right)\right)$. If the Hessian matrix is positive definite, then it is a minimisation problem. Since we have got two decision variables $\left(x_{i}^{*}, i=1,2\right)$, then the Hessian matrix is given as follows:

$$
H\left(X^{*}\right)=\left(\begin{array}{cc}
\frac{\partial^{2} C}{\partial x_{1}^{* 2}} & \frac{\partial^{2} C}{\partial x_{1}^{*} \partial x_{2}^{*}} \\
\frac{\partial^{2} C}{\partial x_{2}^{*} \partial x_{2}^{*}} & \frac{\partial^{2} C}{\partial x_{2}^{* 2}}
\end{array}\right)
$$

We now consider the Lagrangian function because it is a constrained optimisation problem. That is,

$$
\begin{aligned}
L= & h_{1}\left(x_{1}-x_{1}^{*}\right)^{2}+\mu_{1}\left(c_{1}-\mu\right)+h_{2}\left(x_{2}-x_{2}^{*}\right)^{2} \\
& +\mu_{2}\left(c_{2}-\mu\right)-\lambda_{1}\left(x_{1}^{*}-x_{1}+x_{12}-\mu_{1}+D_{1}\right) \\
& -\lambda_{2}\left(x_{2}^{*}-x_{2}-\mu_{2}+D_{2}\right) \\
& -\lambda_{3}\left[x_{1} x_{2}\left(x_{1}+x_{1}^{*}\right)\left(x_{2}+x_{2}^{*}\right)-\frac{D_{1} D_{2}}{\mu^{2}}\right] \\
& +h_{2} x_{21}-\lambda_{4}\left(x_{1}^{*}-x_{2}^{*}\right)-\lambda_{5}\left(x_{1}^{*}+x_{2}^{*}-H\right) \\
& -\lambda_{6}\left(x_{1}^{*}+x_{2}^{*}-I_{\min }\right) .
\end{aligned}
$$

Taking note that it is not possible to issue out product 2 in the absence of product 1 . The first and second derivatives can now be worked out and they are as follows:

$$
\begin{aligned}
\frac{\partial L}{\partial x_{1}^{*}}= & -2 h_{1}\left(x_{1}-x_{1}^{*}\right)-\lambda_{1}-\lambda_{3} x_{1} x_{2}\left(x_{2}+x_{2}^{*}\right) \\
& -\lambda_{4}-\lambda_{5}-\lambda_{6}, \\
\Longrightarrow & \frac{\partial^{2} L}{\partial x_{1}^{* 2}}=2 h_{1}, \quad \frac{\partial^{2} L}{\partial x_{1}^{*} \partial x_{2}^{*}}=-\lambda_{3} x_{1} x_{2}, \\
\frac{\partial L}{\partial x_{2}^{*}}= & -2 h_{2}\left(x_{2}-x_{2}^{*}\right)-\lambda_{2} \\
& -\lambda_{3} x_{1} x_{2}\left(x_{1}+x_{1}^{*}\right)-\lambda_{4}-\lambda_{5}-\lambda_{6}, \\
\Longrightarrow & \frac{\partial^{2} L}{\partial x_{2}^{* 2}}=2 h_{2}, \quad \frac{\partial^{2} L}{\partial x_{2}^{*} \partial x_{1}^{*}}=-\lambda_{3} x_{1} x_{2} .
\end{aligned}
$$

Substituting the derivatives into the Hessian matrix gives

$$
H\left(X^{*}\right)=\left(\begin{array}{cc}
2 h_{1} & -\lambda_{3} x_{1} x_{2} \\
-\lambda_{3} x_{1} x_{2} & 2 h_{2}
\end{array}\right)
$$

When $k=1$, the principal minor determinant of $H\left(X^{*}\right)$ is $\operatorname{det}\left(2 h_{1}\right)=2 h_{1}>0$, which is positive. When $k=2$, the principlal minor determinant is

$$
\left|\begin{array}{cc}
2 h_{1} & -\lambda_{3} x_{1} x_{2} \\
-\lambda_{3} x_{1} x_{2} & 2 h_{2}
\end{array}\right|=4 h_{1} h_{2}-\left(\lambda_{3} x_{1} x_{2}\right)^{2}>0 .
$$

The principal minor determinant is also positive meaning to say that all principal minor determinants are positive for all $k$. Thus, the Hessian matrix is positive definite. This implies that the optimal solution to our nonlinear inventory programming model would be a minimum. As a result, the problem we have formulated is a minimisation problem.

\section{Conclusion}

We can conclude that a simple mathematical programming model for a single continuous review period can be used to minimise the total inventory costs for the two products. It is possible to come up with an optimal ordering policy by applying mathematical programming modeling. Thus, necessary and sufficient conditions for optimality are satisfied; hence, a generalised optimal joint ordering policy can be obtained. Having appreciated that a two-product inventory system with a joint ordering policy can be formulated into a mathematical programming model, then further research can be done on exploring heuristics suitable in deriving an optimal solution. The joint ordering policy can be advanced to at least three products whilst allowing issuing out of any other product in the absence of any other product under consideration. Factors like issuing out of a certain quantity of another product (which increases the holding cost), backordering, and so forth can be introduced to make the model more complex. It is important to note that we can also use either collected or simulated data to validate the model. 


\section{References}

[1] V. S. S. Yadavalli and J. W. Jourbet, "A two-product single period manufacturing and supply system," Journal of the Southern African Institute for Management Scientist, vol. 12, no. 2, pp. 3439, 2003.

[2] E. Mohebbi and M. J. M. Posner, "Multiple replenishment orders in a continuous-review inventory system with lost sales," Operations Research Letters, vol. 30, no. 2, pp. 117-129, 2002.

[3] E. Mehdizadeh and M. J. Tarokh, "An integrated JIT inventory model for supply chain management: single supplier-single buyer with multiple products," in Proceedings of the IEEE International Conference on Service Operations and Logistics, and Informatics (SOLI '06), pp. 288-293, June 2006.

[4] V. S. S. Yadavalli, C. de w van Schoor, and S. Udayabaskaran, "A substitutable two-product inventory system with jointordering policy and common demand," Applied Mathematics and Computation, vol. 172, no. 2, pp. 1257-1271, 2006.

[5] A. A. Taleizadeh, H. Moghadasi, S. T. A. Niaki, and A. Eftekhari, "An economic order quantity under joint replenishment policy to supply expensive imported raw materials with payment in advance," Journal of Applied Sciences, vol. 8, no. 23, pp. 42634273, 2008 .

[6] Y.-C. Chiou and L. W. Lan, "Ordering and warehousing strategies for multi-item multi-branch firm's inventory: clustering approaches," Journal of the Eastern Asia Society for Transportation Studies, vol. 6, pp. 2809-2821, 2005.

[7] B. Roushdy, N. Sobhy, A. Abdelhamid, and A. Mahmoud, "Inventory control for a joint replenishment problem with stochastic demand," World Academy of Science, Engineering and Technology, vol. 26, pp. 156-160, 2011. 


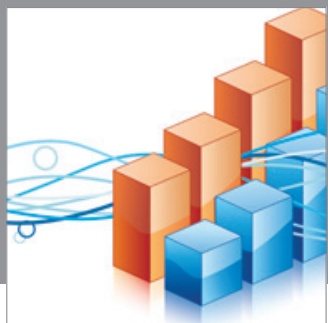

Advances in

Operations Research

mansans

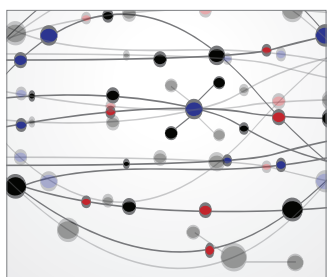

The Scientific World Journal
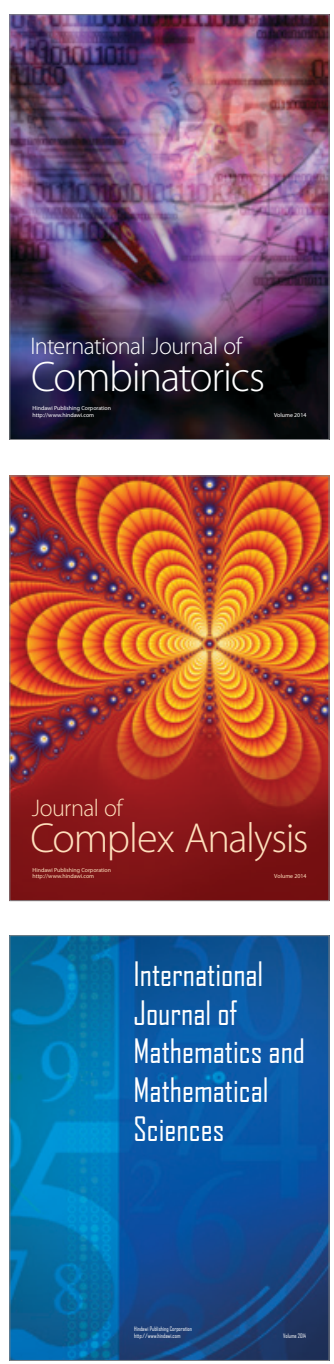
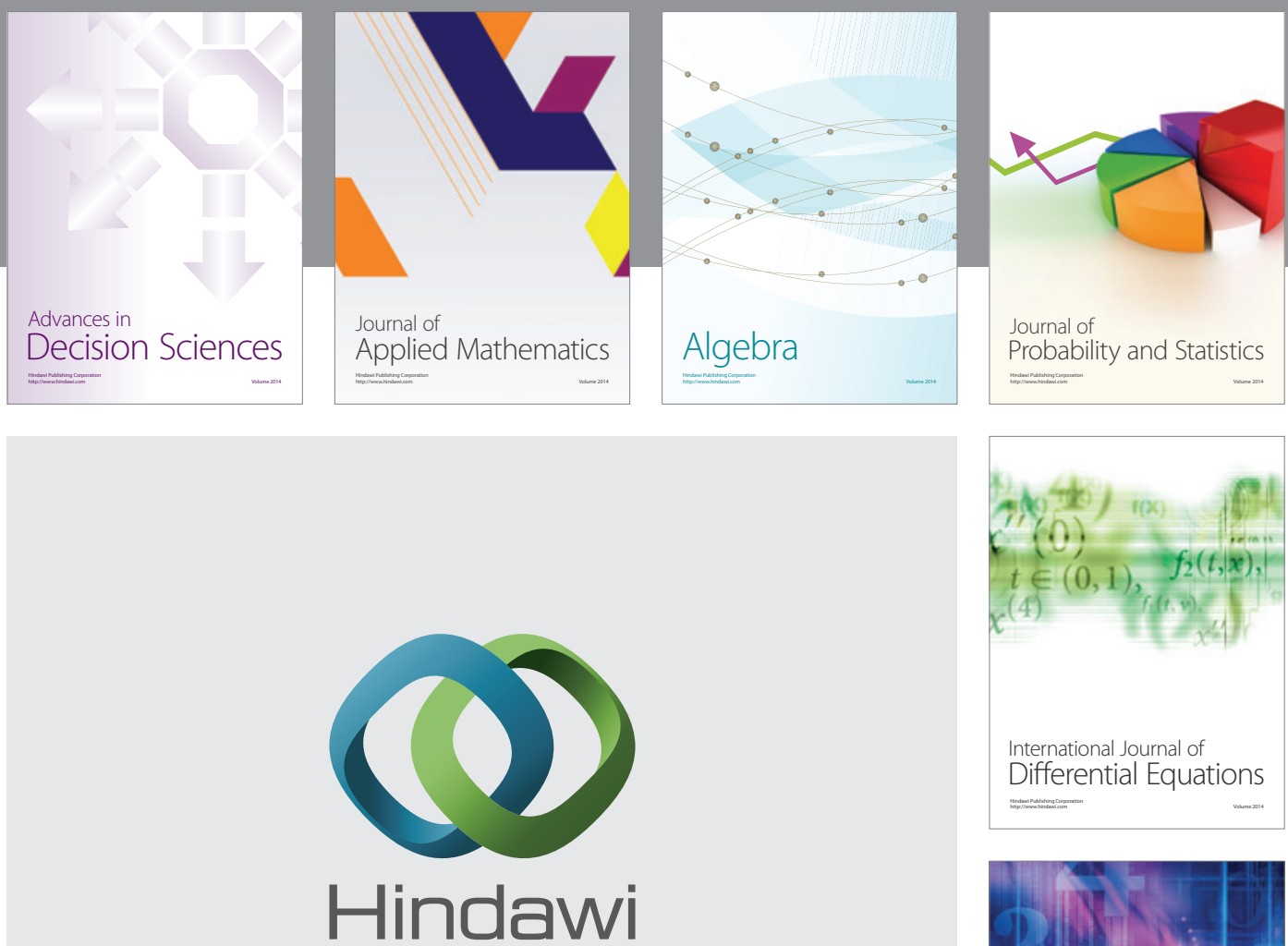

Submit your manuscripts at http://www.hindawi.com
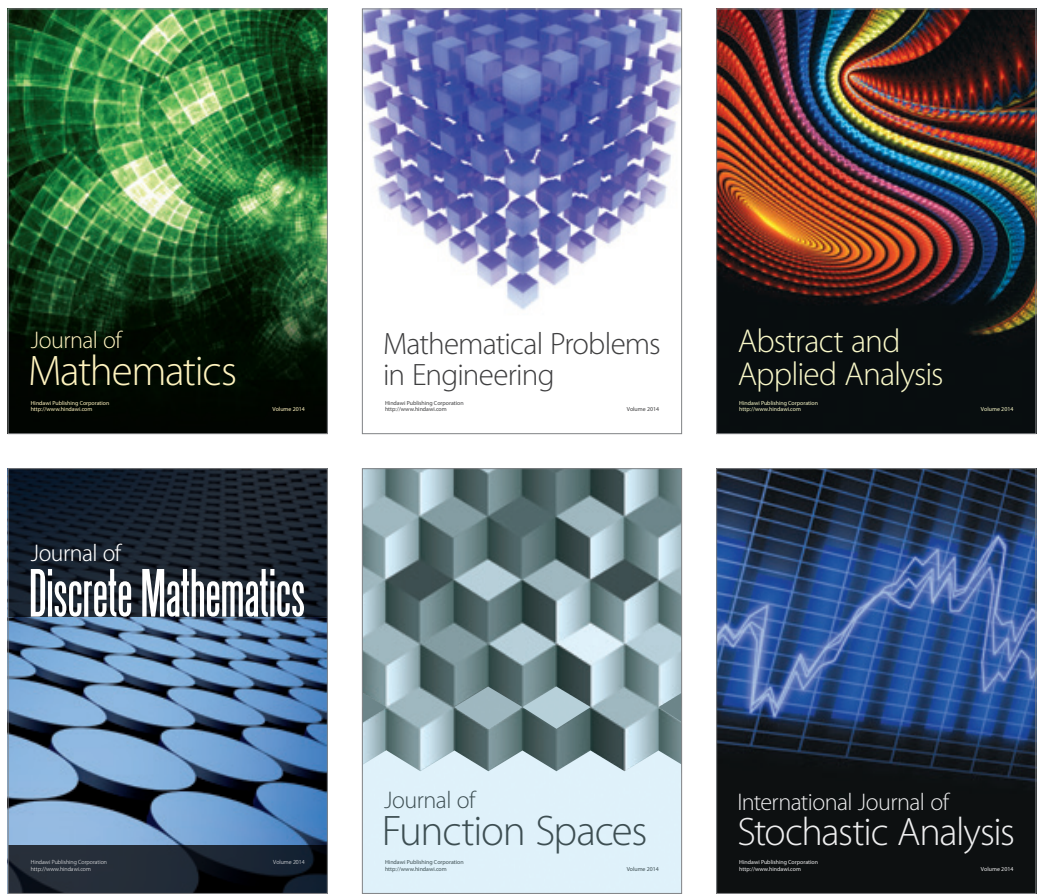

Journal of

Function Spaces

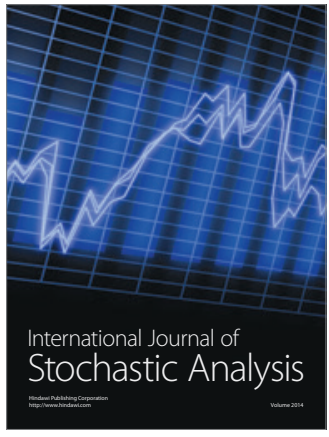

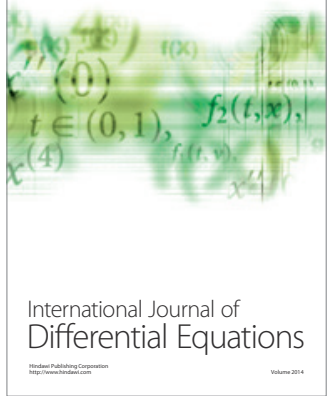
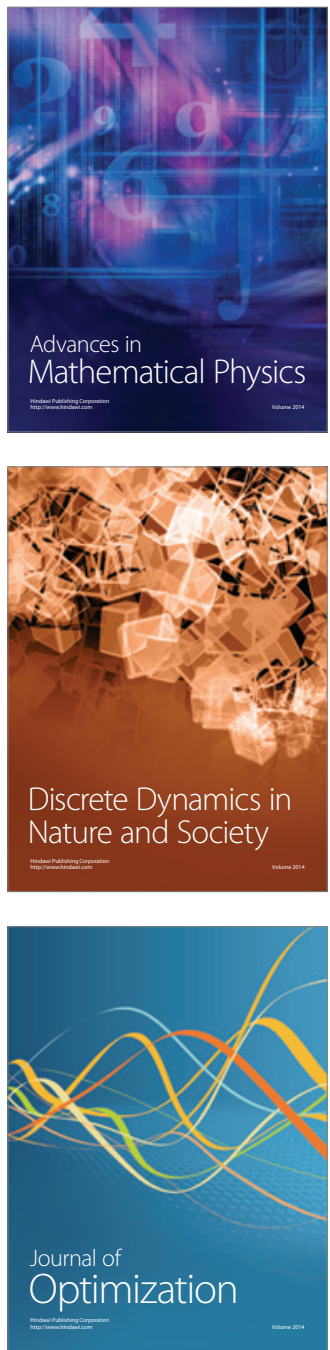